\title{
Variabilité agro-hydrologique des cultures de décrue. Une étude de cas dans la moyenne vallée du fleuve Sénégal
}

\author{
Jean-Christophe Poussin ${ }^{1, *}$, Didier Martin ${ }^{1}$, Jean-Claude Bader ${ }^{1}$, Djiby Dia ${ }^{2}$, Sidy Mohamed Seck ${ }^{3}$ \\ et Andrew Ogilvie ${ }^{4}$ \\ ${ }^{1}$ Institut de recherche pour le développement (IRD), UMR G-EAU, 34394 Montpellier, France \\ 2 Institut sénégalais de recherche agricole (ISRA), Bureau d'analyses macro-économiques, Dakar, Sénégal \\ ${ }^{3}$ Université Gaston Berger, Saint-Louis, Sénégal \\ ${ }^{4}$ Institut de recherche pour le développement (IRD), UMR G-EAU, Dakar, Sénégal
}

\begin{abstract}
Résumé - Après l'édification des barrages sur le fleuve Sénégal, l'agriculture irriguée devait progressivement remplacer l'agriculture de décrue dans la moyenne vallée. Néanmoins, lorsque la crue est d'ampleur suffisante, on observe encore des cultures de décrue dans le lit majeur. Cette étude, réalisée entre juin 2016 et décembre 2019 dans la cuvette de Podor (Sénégal), vise à évaluer la surface inondée et celle cultivée en décrue, à caractériser les producteurs et à estimer les productions. Sur quatre crues observées, trois ont été suffisantes pour inonder plus de 1000 ha et exploiter plus de 400 ha, avec principalement du sorgho associé au niébé. Les crues actuelles sont moins fortes qu'avant 1976, mais l'exondation de la zone exploitable est plus précoce et la période propice aux semis est relativement plus longue. La cuvette est exploitée essentiellement par des habitants de la ville de Podor, agriculteurs ou non, irrigants ou non. La production en 2018 a été estimée à 121 tonnes de grains de sorgho et niébé, et 117 tonnes de fourrage pour 470 ha implantés. C'est peu et aléatoire comparé aux cultures irriguées, mais sans investissement, et les résidus de culture sont d'excellents fourrages. L'agriculture de décrue peut donc être une activité complémentaire rentable pour la population locale. Elle pourrait aussi être rentable à l'intérieur des périmètres irrigués de la moyenne vallée, qui sont inondables et ne sont donc exploités, au mieux, qu'une fois par an seulement. Déjà contrôlé à $50 \%$ depuis 1987, le débit du fleuve pourrait être régulé à $70 \%$ par des barrages réservoirs dans un proche avenir. Le soutien de crue deviendra alors indispensable, malgré son impact négatif sur la production électrique, si l'on veut préserver des cultures de décrue ou certains services écosystémiques nés de l'inondation dans la vallée.
\end{abstract}

Mots clés : agriculture de décrue / inondation / barrage / sorgho / fourrage

\begin{abstract}
Agro-hydrological variability of flood recession crops. A case study in the Middle Valley of the Senegal River. After the construction of dams on the Senegal River, irrigated agriculture was to gradually replace recession agriculture in the middle valley. Nevertheless, when the flood magnitude is sufficient, flood recession crops are still sown in the alluvial plain inside depressions called "cuvettes". This study, carried out between June 2016 and December 2019 in the Podor cuvette, aimed to assess the flooded and cultivated area with flood-recession crops, characterize producers and estimate productions. Out of four floods observed, three were sufficient to flood more than 1000 ha and exploit more than 400 ha with mainly sorghum associated with cowpea. The present floods are lower than before 1976, but the water withdraws earlier and the planting period is longer. The cuvette is exploited mainly by inhabitants of the Podor town, farmers or not, irrigators or not. Production in 2018 has been estimated at 121 tons of sorghum and cowpea grains, and 117 tons of fodder for 470 ha planted. This production is weak and erratic compared to irrigated crops, but requires no investment and crop residues provide excellent fodders. Flood recession agriculture can therefore be a profitable complementary activity for the local population. It could also be profitable within irrigated schemes in the middle valley that are floodable and therefore exploited only once a year. $50 \%$ of river flows are regulated since 1987 and this could increase to $70 \%$ in the near future following the
\end{abstract}

\footnotetext{
*Auteur de correspondance : poussin@ird.fr
} 
construction of additional reservoir dams. Flood support will then become essential, despite its negative impact on hydroelectric production, if recession crops or ecosystem services sustained by flooding in the valley are to be preserved.

Keywords: flood recession agriculture / flooding / dam / sorghum grain / fodder

\section{Introduction}

Les plaines alluviales des grands fleuves d'Afrique subsaharienne sont traditionnellement exploitées après l'inondation (Adams, 1993). L'agriculture traditionnelle de décrue consiste à implanter, par semis ou repiquage, une culture, généralement du sorgho, après la décrue afin qu'elle exploite l'humidité du sol. C'est une culture extensive, à faible densité de plantation et sans ou très faible apport d'engrais, et pour laquelle la surface exploitable varie d'une année à l'autre selon l'ampleur et la durée de l'inondation (Boutillier et al., 1962; Lericollais et Schmitz, 1984; Comas et Gómez MacPherson, 2002; Sidibé et al., 2016).

Les barrages, qui sont généralement édifiés pour la production électrique et/ou le soutien du débit d'étiage avec le développement de l'irrigation, réduisent l'ampleur des crues et par conséquent les surfaces exploitables en décrue (Bader et al., 2003). C'est le cas du fleuve Sénégal, sur lequel l'Organisation pour la mise en valeur du fleuve Sénégal (OMVS) a édifié le barrage anti-sel de Diama en 1986, à proximité de l'embouchure, puis le barrage-réservoir de Manantali en 1987, dans le bassin amont. L'objectif de ces barrages est à la fois la production hydroélectrique et le développement de l'irrigation et de la navigation, tout en soutenant la crue pour maintenir au moins 50000 ha exploitables en décrue ainsi que de nombreux autres services écosystémiques liés à l'inondation dans la "moyenne vallée», entre Matam et Dagana (SCP et al., 2009). Néanmoins, l'agriculture irriguée a vocation à remplacer progressivement l'agriculture traditionnelle de décrue (Boutillier et Schmitz, 1987).

L'agriculture de décrue dans la moyenne vallée du fleuve Sénégal est un pilier du système de production traditionnel (Boutillier et al., 1962), car la récolte a lieu en saison sèche et la production contribue ainsi à l'alimentation des populations et des troupeaux en période de soudure. Avec la faible ampleur des crues entre 1976 et 1993, les surfaces exploitables en décrue ont été beaucoup plus faibles et certaines ont été aménagées pour développer l'agriculture irriguée. Toutefois, depuis 1994, la crue du fleuve redevient suffisante certaines années pour inonder la vallée et l'agriculture de décrue persiste (Le Roy, 2008; Bruckmann, 2016).

Cette étude, réalisée de juillet 2016 à décembre 2019, s'intéresse à la « cuvette» de Podor. Cette cuvette a été choisie car elle bénéficie de connaissances antérieures sur son fonctionnement agro-hydrologique en tant qu'un des sites suivis par le Projet d'optimisation de la gestion des réservoirs (POGR) de l'OMVS, visant pour partie à mesurer l'influence de la gestion des barrages sur les cuvettes de décrue (Lamagat, 2001). Elle est également accessible par la route pendant la crue et sa topographie précise est connue. L'objectif est ici de quantifier la surface inondée par la crue et celle cultivée en décrue, d'en estimer les productions, et de caractériser les producteurs. Cette cuvette illustre des caractéristiques des cultures de décrue dans la moyenne vallée du fleuve Sénégal, mais il ne s'agit ni d'extrapoler à l'ensemble de la plaine inondable, ni d'évaluer la place de l'agriculture de décrue dans l'économie locale.

\section{Matériel et méthodes}

\subsection{La cuvette de Podor}

Le lit majeur du fleuve Sénégal dans sa moyenne vallée est extrêmement plat (pentes $<1 \%$ ), avec de légères dépressions (échelle décimétrique) qui forment des défluents et des cuvettes, et son hydrologie est extrêmement complexe en période d'inondation et d'exondation. Issus de la sédimentation des eaux du fleuve, les sols sont composés d'un horizon plus ou moins argileux (de 30 à $60 \%$ d'argile), d'épaisseur variable ( $60 \mathrm{~cm}$ à quelques mètres) et reposant sur un horizon sableux (Maymard, 1960; Boivin et al., 1995).

La cuvette de Podor (Fig. 1) se situe dans un méandre du fleuve, à l'extrême nord du Sénégal et à $180 \mathrm{~km}$ de Saint-Louis. La zone inondée (un maximum de 1960 ha délimités par le POGR) ressemble à un trapèze dont la plus petite base est au nord et la plus grande au sud; sa moitié ouest est pour partie aménagée en périmètres irrigués, partiellement ou totalement inondés lors des crues; la ville de Podor (12 000 habitants en 2007) longe son côté est.

\subsection{Dispositif hydrologique et topographie}

L'exploitation en décrue dépend directement de la crue du fleuve, de sa propagation dans le lit majeur, et de la durée de l'inondation; il est donc essentiel de s'intéresser à l'hydrologie.

Une échelle limnimétrique, calée en altitude absolue (niveau général, $\mathrm{NG}$ ), est installée sur le quai du fleuve à Podor. La cote du fleuve est transmise à l'OMVS deux fois par jour. Ces données, disponibles depuis 1903, sont utilisées ici pour reproduire le niveau et la surface inondée dans la cuvette de Podor avec les modèles de propagation de Lamagat (2001) et Bader et al. (2017).

Installé en 2017 dans une zone basse de la cuvette de Podor et calé en altitude NG, un limnigraphe à pression (1 mesure/ heure) a permis de suivre la cote de l'inondation. De plus, la Coopération japonaise (JICA) nous a fourni un modèle numérique de terrain (MNT) de forte résolution (maille de 2,5 $\mathrm{m}$ et précision centimétrique en altitude) réalisé en 2013 sur l'ensemble de la zone délimitée par le POGR et visant son aménagement pour l'irrigation. Nous avons effectué des levés topographiques (lunette et mires, et GPS différentiel) afin de recaler ce MNT en altitude NG. Ce MNT permet de situer précisément les zones inondées selon la cote de l'inondation mesurée par le limnigraphe. La surface inondée en 2016 et sa cote ont été estimées sur la base d'images Sentinel-2 et de ce MNT. Les crues et surfaces inondées observées entre 2016 et 


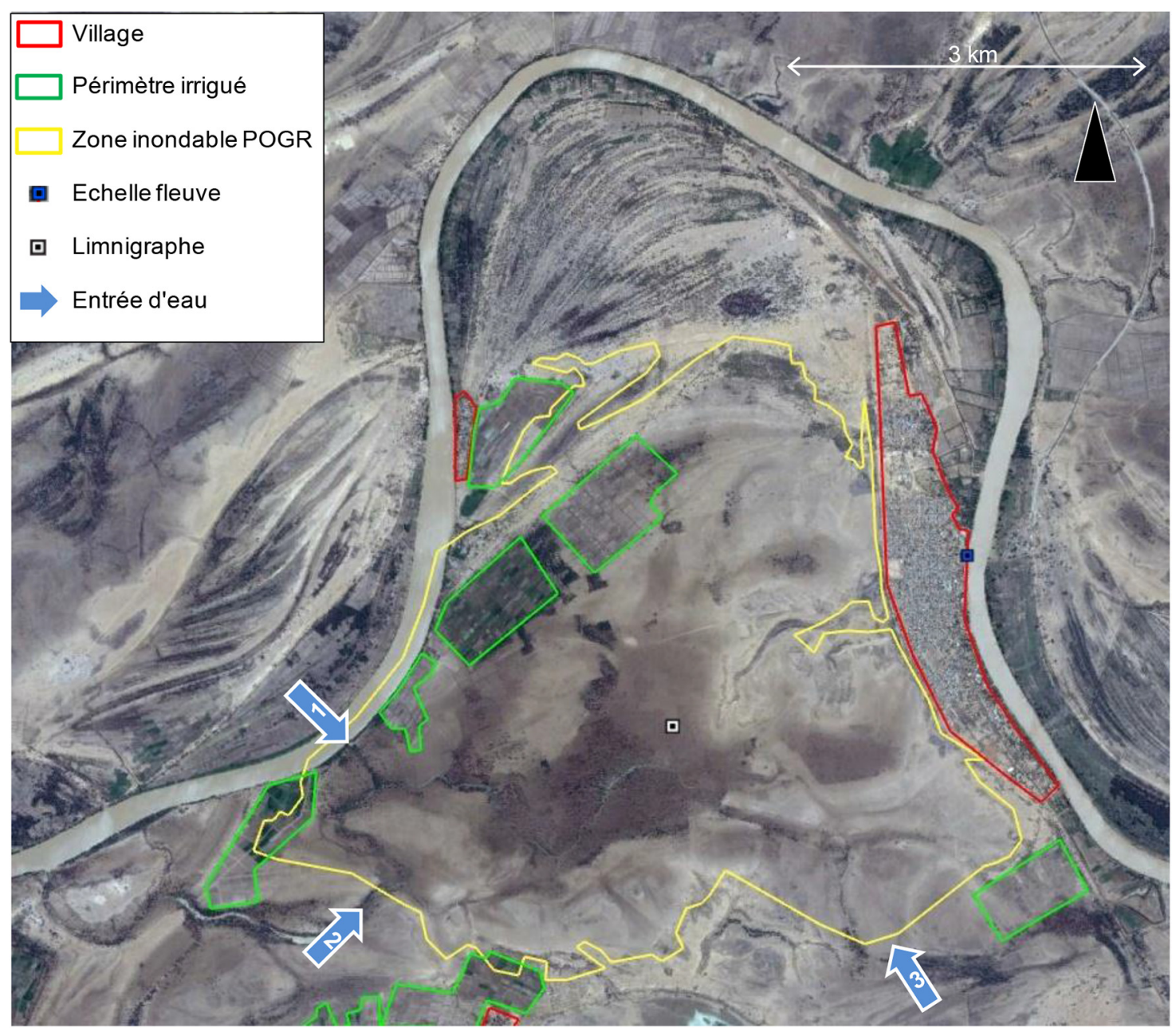

Fig. 1. Carte de la cuvette de Podor indiquant la zone inondable délimitée par le POGR, les villages (Podor à l'est, Ngawlé à l'ouest), les périmètres irrigués, la position de l'échelle limnimétrique du fleuve et du limnigraphe dans la cuvette, et les trois points d'entrée de l'eau (fond de carte: image satellite Google Earth de juillet 2013).

Fig. 1. Map of the Podor cuvette showing the floodplain delimited by the POGR, the villages (Podor in the east, Ngawle in the west), the irrigation schemes, the position of the staff gauge in the river and the limnigraph in the cuvette, and the three water entry points (background map: Google Earth satellite image of July 2013).

2019 ont été comparées aux crues enregistrées par le passé et aux surfaces reproduites par modélisation.

L'existence d'un MNT sur la cuvette de Podor a été un atout essentiel pour cette étude qu'il serait intéressant d'élargir à l'ensemble de la moyenne vallée. La JICA a réalisé pour l'Agence nationale de l'aménagement du territoire une topographie numérique de la vallée du fleuve Sénégal, de Bakel à Saint-Louis (JICA, 2013), mais les MNT construits ne sont pas disponibles.

\subsection{Reconstitution des superficies exploitables en décrue sur la base de la chronique des crues à Podor}

Déjà utilisé par Lamagat (2001) et Bader et al. (2017), puis recalé ici sur toutes les données disponibles, le modèle de propagation reproduit le niveau du plan d'eau dans la cuvette à partir des cotes du fleuve à Podor $(10,3 \mathrm{~cm}$ d'erreur-type et coefficient d'efficacité de Nash et Sutcliffe égal à 0,985 , pour
490 cotes observées pendant 7 crues : 1997 à 2000 et 2017 à 2019). La superficie inondée est ensuite estimée à l'aide de la relation établie par Lamagat (2001) et révisée par Bader (2014).

Les chroniques de cote et de surface du plan d'eau obtenues sont ensuite utilisées pour reconstituer les surfaces exploitables en décrue de 1903 à 2019, correspondant aux zones situées entre les cotes $290 \mathrm{~cm}$ (seuil d'entrée/sortie de l'eau) et $480 \mathrm{~cm}$ NG (au-delà, le sol est impropre aux cultures) qui ont été submergées et définitivement exondées avant le $1^{\text {er }}$ décembre (un semis tardif de sorgho a un rendement faible). On calcule ainsi chaque année les cotes maximale (Hd) et minimale (Hf) de la zone exploitable, les surfaces inondées correspondantes ( $\mathrm{Sd}$ et $\mathrm{Sf}$ ), et les jours de début (Jd) et de fin (Jf) du retrait de l'eau. La surface exploitable $(\mathrm{S}=\mathrm{Sd}-\mathrm{Sf})$ et la durée de retrait de l'eau $(\mathrm{D}=\mathrm{Jf}-\mathrm{Jd})$ sont nulles si la cote du plan d'eau reste inférieure à $290 \mathrm{~cm}$ ou bien supérieure à $480 \mathrm{~cm}$ jusqu'au $1^{\text {er }}$ décembre. 


\subsection{Observation des pratiques et estimation des productions des cultures de décrue}

Entre 2016 et 2019, nous avons observé 4 cycles de cruedécrue, mais seulement 3 campagnes de culture car la crue de 2017 était trop faible. Nous avons comparé nos observations sur les pratiques culturales aux travaux de Boutillier et al. (1962) et Lericollais et Schmitz (1984), qui dressent une image très précise du système de production agricole dans la moyenne vallée du Sénégal avant 1976.

Deux campagnes de photographies aériennes par drone ont été réalisées sur l'ensemble de la cuvette. Celle de janvier 2017 a permis de tracer le parcellaire cultivé après la décrue de 2016. La seconde, en janvier 2019, a permis en plus de classer les parcelles selon la densité et l'état de végétation des cultures après la décrue de 2018. On a distingué quatre classes: (nul) parcelle implantée mais culture non levée ou avortée; (faible) faible densité de végétation cultivée; (moyen) végétation cultivée assez dense et assez homogène; (fort) végétation cultivée dense et homogène.

La production de la campagne 2018 a été estimée à la récolte dans un échantillon de 5 à 8 parcelles récoltées dans chacune des 3 dernières classes. Ces parcelles étaient pour la plupart cultivées en sorgho et/ou niébé, avec parfois du melon et/ou de la pastèque. Pour le sorgho et le niébé, les quantités de produits (graines) et sous-produits (fanes, tiges, cosses) utilisés comme fourrage ont été estimées par décompte et pesée des récipients et fagots récoltés. Les quantités de fanes de niébé récoltées n'ont pas pu être mesurées; la quantité de fourrage est donc sous estimée. Les produits du sorgho et du niébé ont été regroupés pour effectuer une analyse de variance. Les producteurs ont été interrogés sur les prix des produits et sous-produits, notamment pour les melons et les pastèques.

Enfin, une enquête a été menée à l'implantation des cultures en 2018. Une soixantaine de producteurs ont été interrogés dans leur parcelle (âge, expérience en agriculture de décrue, village d'origine, activité principale) et les profondeurs de semis ont également été mesurées.

\section{Résultats et discussion}

\subsection{Crue du fleuve et surface inondée}

Les observations réalisées entre 2016 et 2019 montrent que lors d'une crue, la cuvette de Podor est alimentée d'abord par l'ouest, puis par le sud-ouest, et enfin par le sud (flèches 1, 2 et 3 sur la Fig. 1). Le limnigramme de l'inondation est légèrement en retard et moins élevé que l'hydrogramme du fleuve à cause des seuils à franchir et du temps de propagation de l'eau dans le lit majeur (Fig. 2a). Lors de la décrue, ces mêmes seuils retiennent l'eau dans la cuvette, et la surface qui reste inondée après le 30 novembre est difficilement exploitable (présence de flaques) et très peu productive (Maymard, 1957; Jamin, 1986). En 2017, la crue a été courte et de faible ampleur (cote maximale $321 \mathrm{~cm}$ le 05/09). Les trois autres années, le pic de crue a eu lieu entre le 30/09 (2019) et le 03/10 (2016), avec une cote maximale entre $456 \mathrm{~cm}$ (2019) et $477 \mathrm{~cm} \mathrm{(2016).}$ L'amplitude de la crue a déterminé la cote maximale du plan d'eau dans la cuvette (entre $303 \mathrm{~cm}$ en 2017 et $461 \mathrm{~cm}$ en 2016) et la surface maximale inondée (entre 380 ha en 2017 et
1590 ha en 2016; Fig. 2b). La surface qui est restée inondée après le 30 novembre est de l'ordre de 100 ha.

Les producteurs sèment les cultures dans les zones exondées au rythme de la décrue. Plus le semis est précoce, plus le cycle végétatif du sorgho de décrue - plante photopériodique - est long, et plus le rendement potentiel est élevé (Chantereau et al., 2013). Par conséquent, à l'échelle de cette cuvette, à crue égale de forte ampleur, plus la décrue est précoce et rapide, plus le rendement potentiel est a priori élevé.

Les valeurs modélisées de superficie exploitable en décrue à Podor permettent de distinguer trois périodes avec des crues très différentes: 1903-1975, 1976-1993 et 1994-2019 (Fig. 3). Jusqu'en 1975, les crues entièrement naturelles sont globalement fortes. Puis de 1976 à 1993, les écoulements naturels très faibles (et partiellement stockés de 1987 à 1991 pour remplir Manantali) produisent de faibles crues. Les crues reviennent à un meilleur niveau à partir de 1994. Elles bénéficient d'abord du soutien de crue jusqu'en 2002, puis d'une amélioration des apports naturels sur le haut bassin, qui pallie depuis 2004 la forte régulation des débits liée à la production hydroélectrique. Enfin, le barrage de Diama rehausse le niveau d'étiage depuis 1992.

La figure 3 montre que plus la crue est haute, plus son pic est tardif et sa durée est longue. En plus des différences de superficie exploitable entre les trois périodes (moyenne: 1212, 247 et 856 ha respectivement), on observe des différences sur la période d'exondation de la zone exploitable en cultures de décrue (date moyenne de début d'exondation : 28/10, 24/09 et $02 / 10$; date moyenne de fin: $18 / 11,10 / 10$ et $28 / 10$ ). Ces différences se retrouvent sur les fonctions de répartition fréquentielles de ces variables (Fig. 4). Depuis 1994, la superficie moyenne exploitable en décrue à Podor a diminué de $30 \%$ par rapport à celle d'avant 1976 . En revanche, le retrait des eaux est beaucoup plus précoce, ce qui est a priori favorable aux productions.

\subsection{Les producteurs de décrue et leurs pratiques culturales}

Sur les 60 paysans interrogés en 2018, 50 dont 12 femmes étaient les responsables des parcelles; 54 venaient de Podor, 4 de Guia, au bord du Doué, et 2 de Taredji, au bord de la zone dunaire. Deux seulement avaient moins de 30 ans et quasiment tous déclaraient être assez ou très expérimentés en agriculture de décrue. Leurs activités principales étaient très diverses: $25 \%$ pratiquaient l'agriculture non irriguée et $20 \%$ l'agriculture irriguée, $25 \%$ étaient artisans ou commerçants (en activité ou retraités), $20 \%$ étaient salariés (en activité ou retraités), et enfin, l'un était marabout, un autre musicien, et une autre femme au foyer. La proximité de la ville de Podor explique sans doute la forte proportion de «non-agriculteurs» et montre aussi que l'agriculture de décrue peut intéresser une collection hétérogène d'habitants.

Les producteurs attendent 3 à 10 jours après l'exondation afin que le sol soit portant (une croûte sèche se forme en surface). Avant le semis, ils suppriment les arbustes et enlèvent les mauvaises herbes ou réalisent parfois un sarclage ou grattage du sol en culture attelée asine ou équine, voire bovine (Fig. 5). Au semis, ils font des trous espacés d'environ $90 \mathrm{~cm}$ à l'aide d'une houe pour casser la croûte de sol en surface, puis d'un bâton plantoir, et placent jusqu'à plus d'une dizaine de 
a)

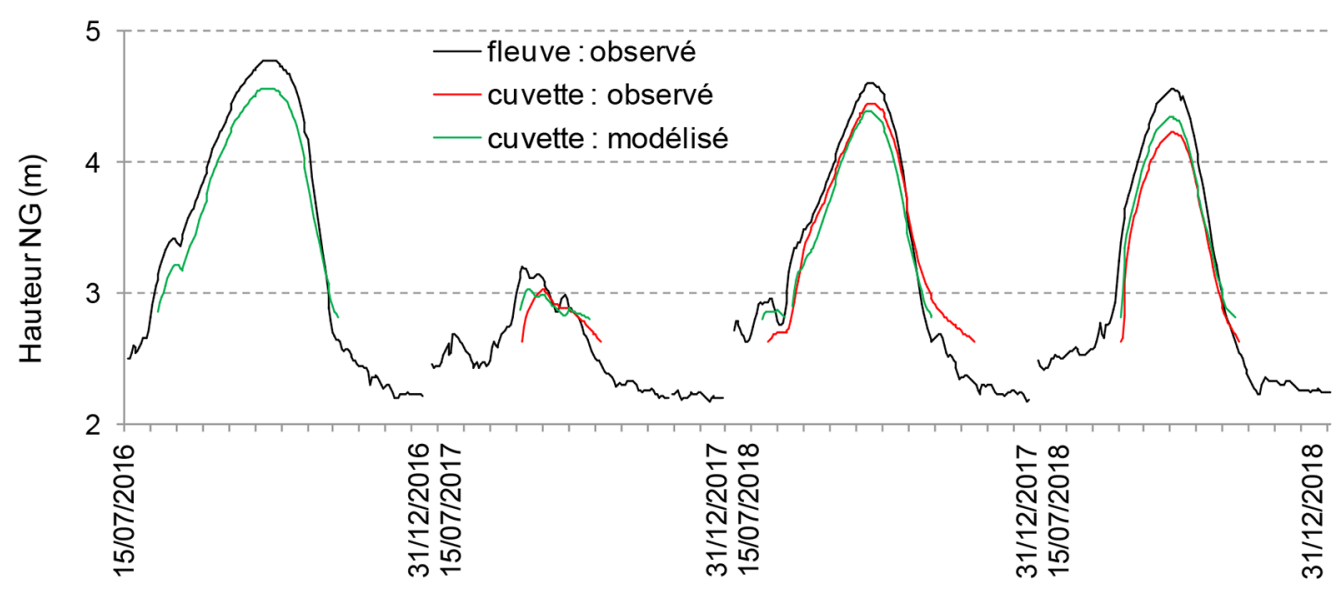

b)

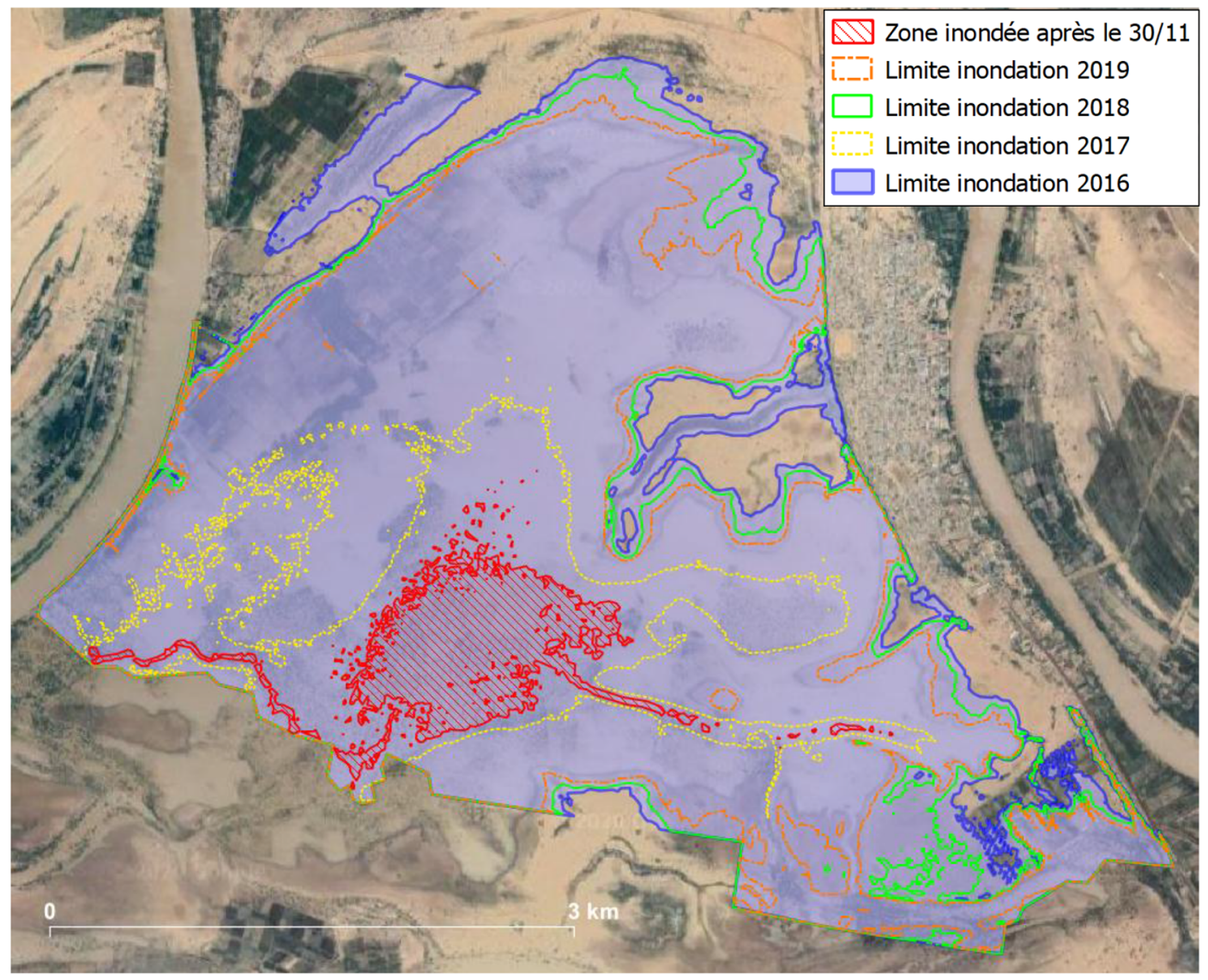

Fig. 2. (a) Cotes du fleuve à Podor et cotes, observées et modélisées, dans la cuvette entre le 15 juillet et le 31 décembre, et (b) surface maximale inondée en 2016, 2017, 2018 et 2019, et surface inondée après le 30 novembre dans la zone délimitée par le POGR.

Fig. 2. (a) River elevation at Podor and water level, observed and modelled, in the cuvette between 15th July and 31st December, and (b) maximum flooded area in 2016, 2017, 2018 and 2019, and flooded area after 30th November in the area delimited by the POGR.

semences, souvent traitées à l'insecticide (fourni gratuitement par les services agricoles de l'État), au fond des trous (profondeur médiane: $17 \mathrm{~cm}$ ) et les recouvrent de sable pour les protéger des oiseaux. Une ligne de plantation plus dense marque la frontière de la parcelle. Parfois, la plantation se fait en cercles concentriques (Fig. 6) pour contrôler et augmenter la densité. On plante dans le même trou principalement du sorgho et/ou du niébé. Cette plantation est parfois associée à du melon et/ou de la pastèque plantés quelques jours plus tôt et plus espacés (3 à $4 \mathrm{~m}$ ). Après la levée, voire bien après, les producteurs font un sarclage manuel (s'il n'a pas eu lieu avant le semis), accompagné parfois d'un démariage. Après l'épiaison, ils protègent les épis de sorgho des oiseaux en les enroulant dans un bout de tissu ou de sac plastique, et ils pratiquent un gardiennage et/ou installent des épouvantails (drapeaux ou objets ; Fig. 7). La récolte démarre début février par le melon, la pastèque et le niébé, et se termine fin février par le sorgho. Les épis de sorgho et les gousses de niébé sont séchés puis égrainés au champ. Les résidus (tiges et rachis de sorgho, fanes et cosses de niébé) sont utilisés comme fourrage. 


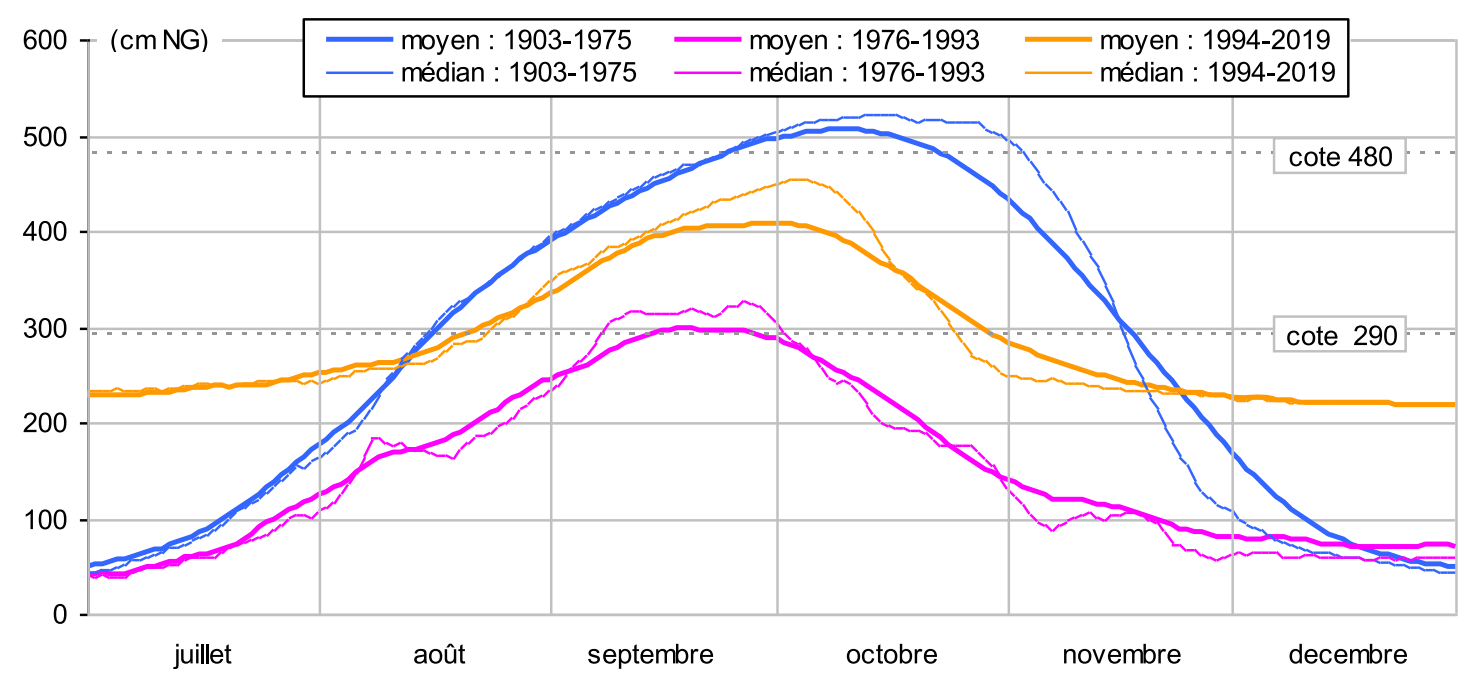

Fig. 3. Limnigrammes moyens et médians observés du fleuve Sénégal à la station de Podor sur les périodes 1903-1975, 1976-1993 et 19942019.

Fig. 3. Mean and median limnograph of the Senegal River observed at the Podor station over the periods 1903-1975, 1976-1993 and 19942019.
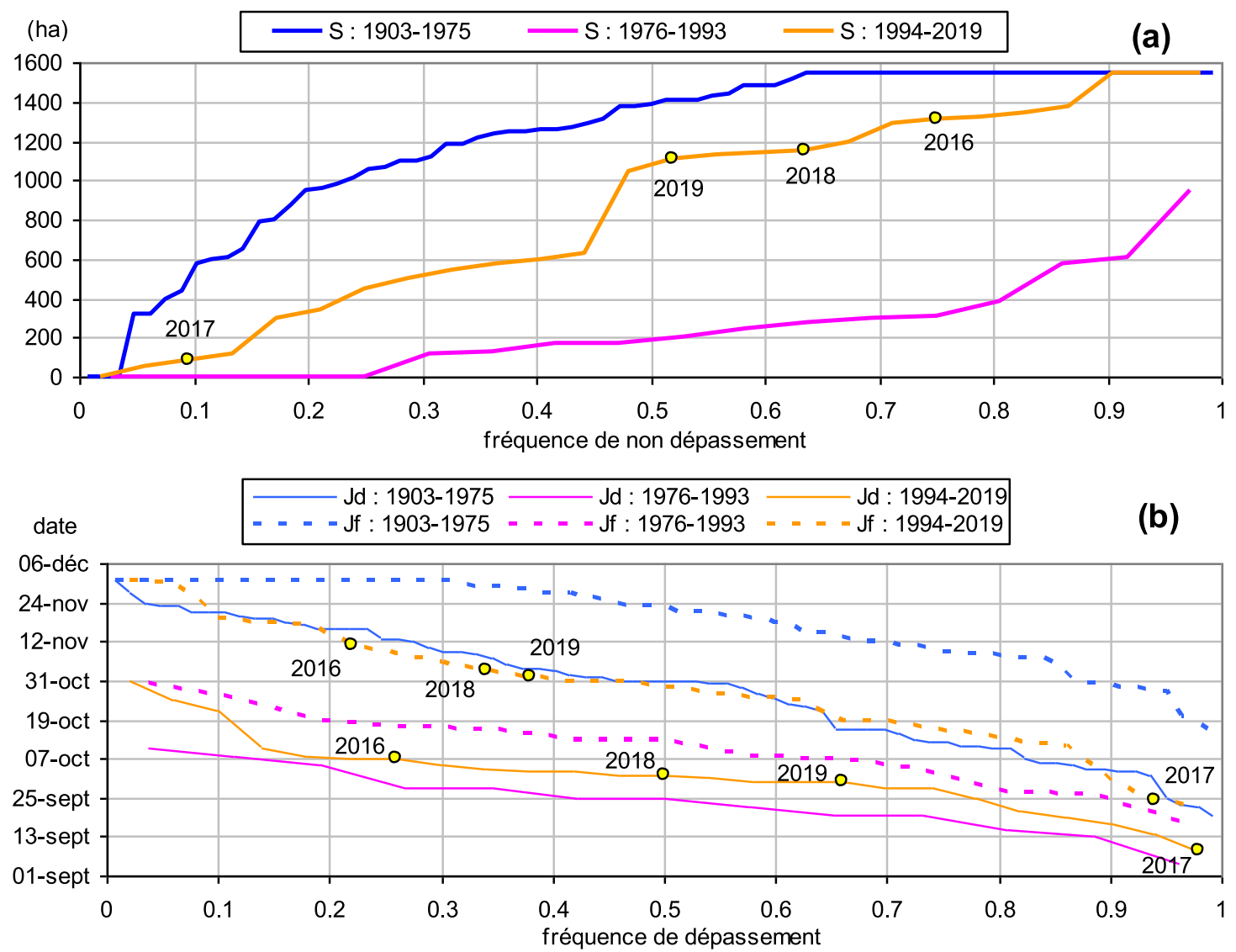

Fig. 4. Fonctions de répartition de la superficie de la zone exploitable (S) et des dates de début (Jd) et de fin (Jf) de retrait des eaux sur cette zone (valeurs modélisées à partir des cotes du fleuve à Podor).

Fig. 4. Distribution functions for the exploitable area (S) and the start (Jd) and end (Jf) dates of water withdrawal from this area (values modelled from river levels at Podor). 


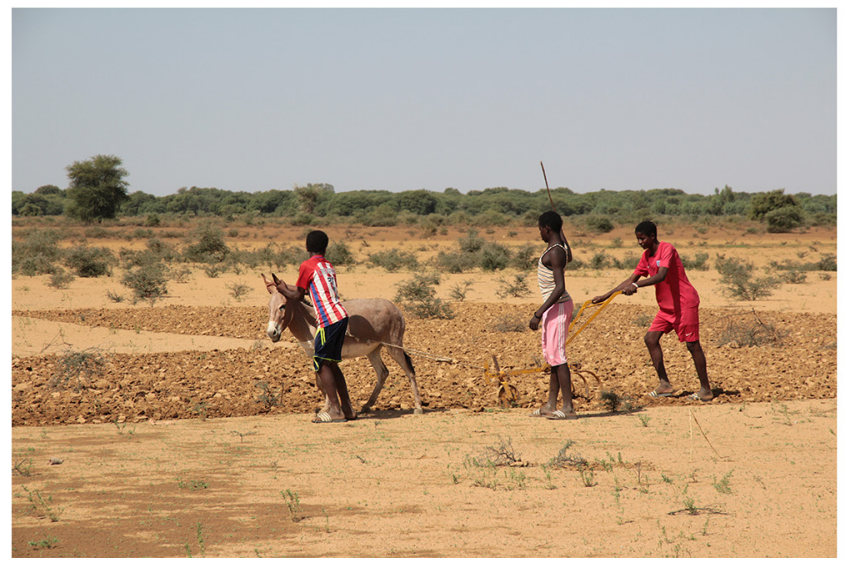

(a)

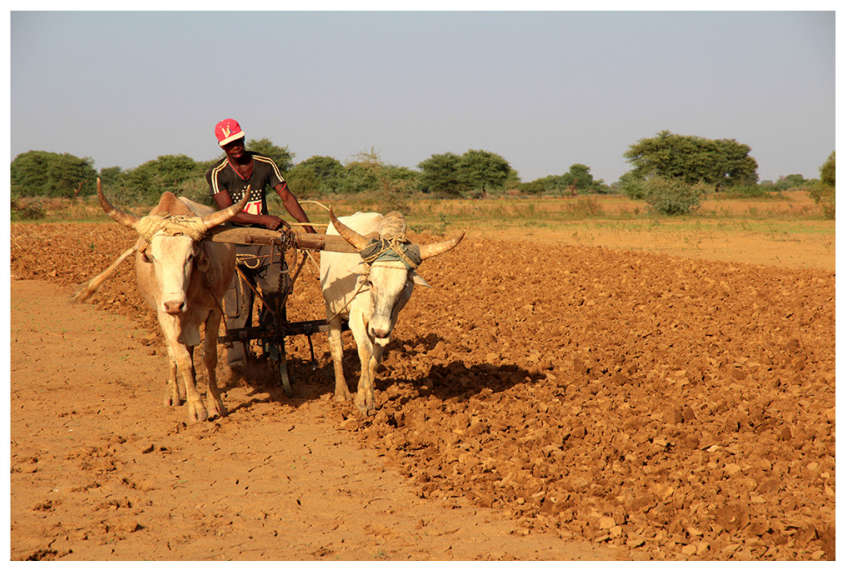

(b)

Fig. 5. Sarclage avant semis (a) en traction asine dans la cuvette de Podor (nov. 2018) et (b) en traction bovine dans la cuvette de Wawa (nov. 2016).

Fig. 5. Weeding prior to sowing (a) in asine traction in the Podor cuvette (Nov. 2018) and (b) in bovine traction in the Wawa cuvette (Nov. 2016).

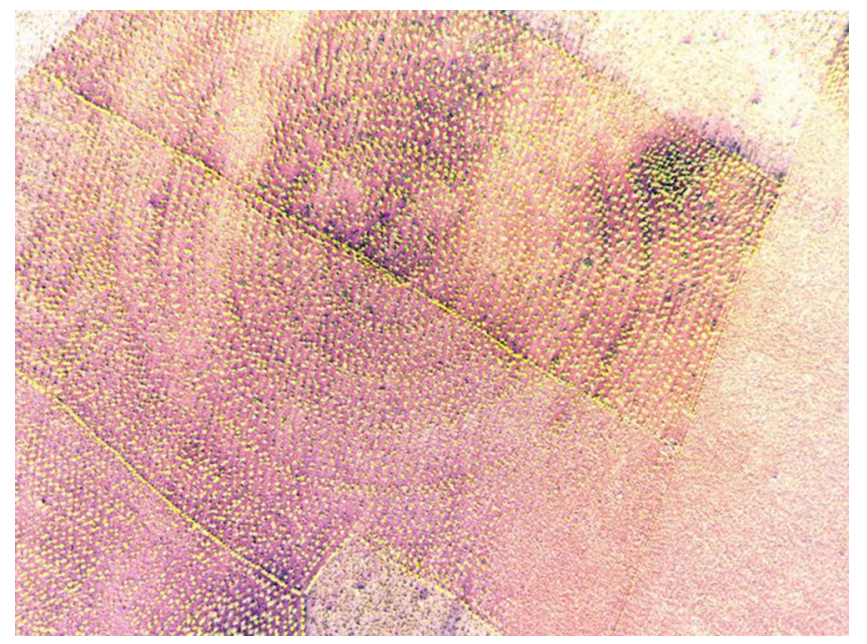

Fig. 6. Photo aérienne d'une plantation de sorgho en cercles concentriques dans la cuvette de Podor (jan. 2017).

Fig. 6. Aerial photo of a sorghum plantation in concentric circles in the Podor cuvette (Jan. 2017).

Après la récolte, il ne reste que les souches de sorgho dans les parcelles (cela permettrait d'estimer la surface récoltée par analyse d'images satellites avant et après récolte).

À l'exception du traitement insecticide des semences, de la plantation circulaire et du sarclage attelé avant semis, ces pratiques sont celles décrites dans les travaux anciens (Boutillier et al., 1962 ; Lericollais et Schmitz, 1984 ; Jamin, 1986). Dans les études récentes, Bruckmann (2016) a aussi observé ce sarclage attelé, mais pas la plantation circulaire. Pourtant, au dire des paysans interrogés, ce sarclage et la plantation circulaire seraient des pratiques traditionnelles. Aujourd'hui, le démariage et le sarclage après la levée sont moins systématiques, de même que le gardiennage, qui n'est plus confié aux enfants, sans doute parce qu'ils sont scolarisés. Observé une seule fois en 2016 dans la cuvette de Podor, le sarclage attelé avant semis était plus fréquent en 2018 et 2019 ; les paysans ont peut-être noté son efficacité sur le contrôle des adventices et un meilleur développement des cultures (effet mulch).

\subsection{Le parcellaire cultivé, les cultures et leur densité de végétation}

Une grande partie de la surface inondable délimitée par le POGR (1960 ha) n'est a priori pas exploitable en décrue car elle est boisée (plus de 300 ha) ou aménagée en périmètres irrigués (plus de 150 ha). Lors de la campagne de décrue 2016, le parcellaire cultivé couvrait 603 ha, soit $53 \%$ de la surface inondée exploitable; en 2018, il couvrait 471 ha, soit $47 \%$ de la surface inondée exploitable. Cette mise en valeur d'environ la moitié de la surface inondée exploitable correspond aux mesures par télédétection faites en 2000 par Mané et Fraval (2001) sur l'ensemble du département de Podor. Lorsque la crue du fleuve dépasse $475 \mathrm{~cm}$, comme en 2016, elle inonde des zones à plus de $460 \mathrm{~cm}$ d'altitude dans la cuvette. Mais les terres situées au-dessus de $480 \mathrm{~cm}$ NG sont impropres aux cultures de décrue car trop sableuses (zones très claires sur le fond de carte de la Fig. 2).

Le contour du parcellaire au nord et à l'est en 2016 et en 2018 suit la limite de la zone inondée (Fig. 8), et les producteurs qui exploitent en bordure se déplacent selon le niveau maximum de l'inondation lorsque celui-ci varie peu, comme entre la crue de 2016 et celle de 2018 (ou de 2019). En 2018, quelques parcelles ont été implantées hors de la zone inondée (Fig. 2 au sud-est), mais abandonnées ensuite. La mise en culture de la zone inondée exploitable est moins systématique au sud et à l'ouest, loin de Podor d'où viennent la majorité des producteurs. Au contraire des observations faites par Saarnak (2003), les villageois de Ngawlé exploitent peu la cuvette de Podor, mais plutôt des jardins et périmètres irrigués maraîchers à la même période. De plus, certaines zones exploitables ne sont pas accessibles car entourées de zones inondées. On note aussi quelques parcelles cultivées dans une partie abandonnée d'un aménagement inondé.

Les producteurs ont implanté majoritairement du sorgho en association avec du niébé quelle que soit l'altitude, et parfois avec du melon et/ou de la pastèque principalement en zone haute (Fig. 9a). Ensuite, loin derrière, ils ont choisi le niébé seul, ou plus rarement et plutôt en zone haute le sorgho seul. 


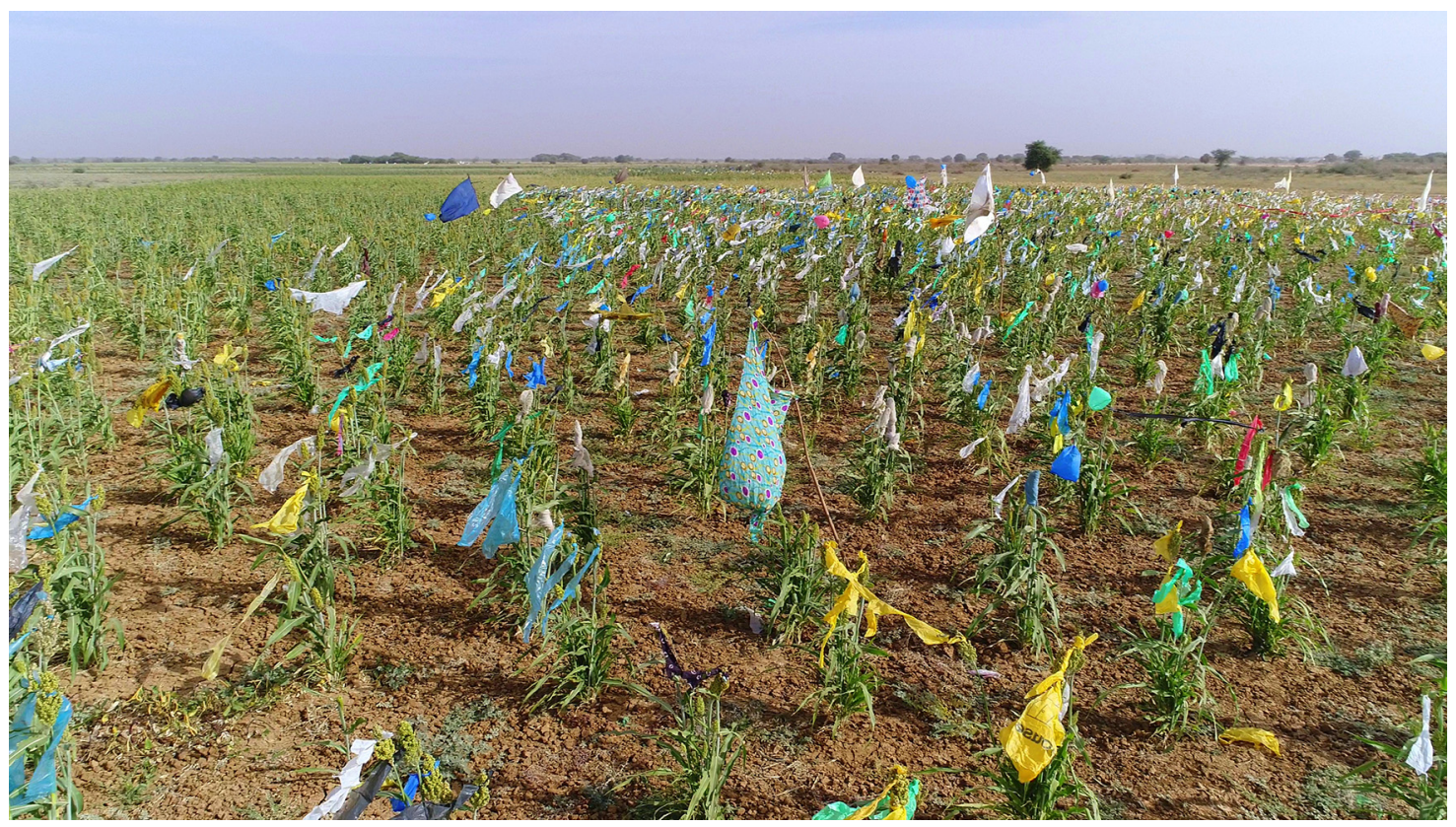

Fig. 7. Protection du sorgho contre les attaques d'oiseaux dans la cuvette de Podor (fév. 2019).

Fig. 7. Protection of sorghum against bird attacks in the Podor cuvette (Feb. 2019).

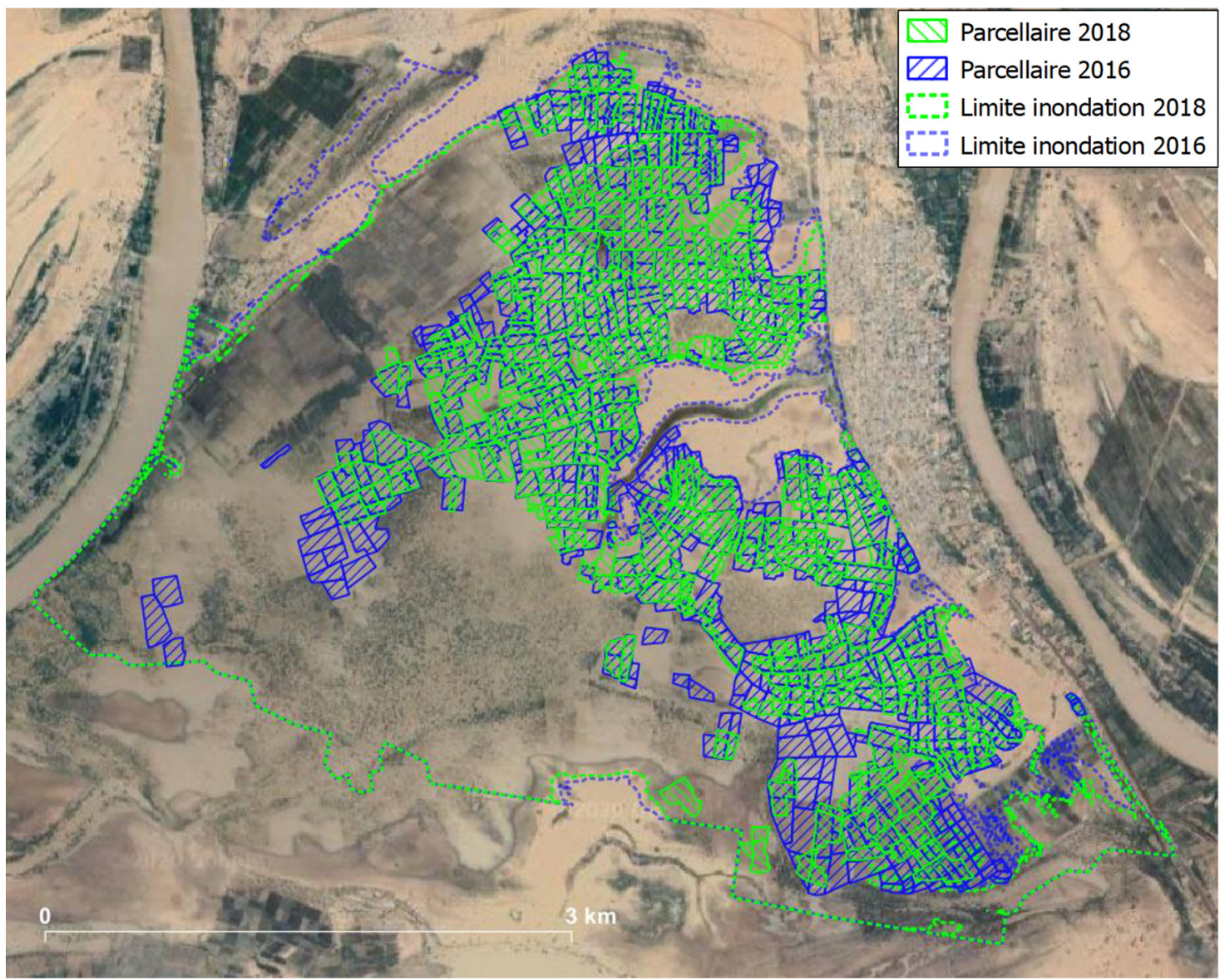

Fig. 8. Limite de l'inondation et parcellaire cultivé en décrue en 2016 et en 2018.

Fig. 8. Flood extent and plots cultivated with flood recession crops in 2016 and 2018. 

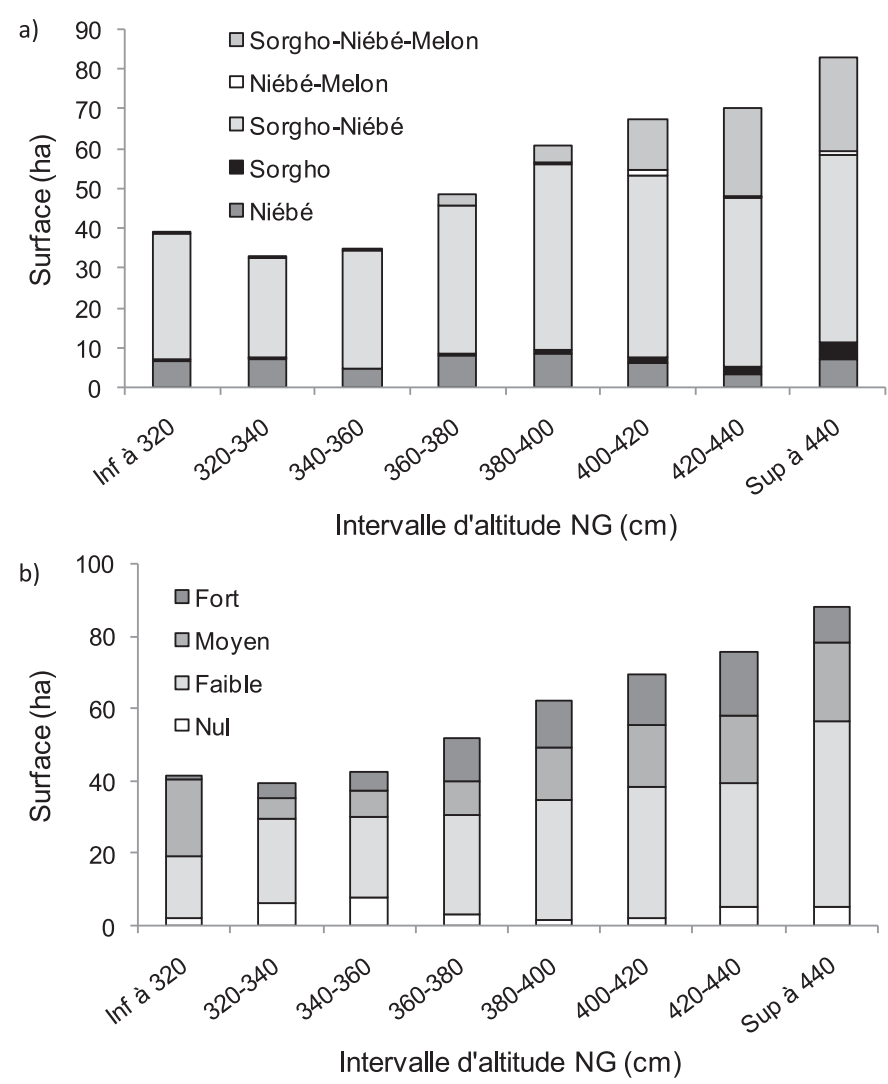

Fig. 9. Répartition de la surface cultivée en 2018 selon l'altitude et (a) la culture, ou (b) la note de densité de végétation.

Fig. 9. Distribution of cultivated area in 2018 according to the altitude and (a) the crop, or (b) the vegetation density score.

Boutillier et al. (1962) ont indiqué que les paysans préféraient cultiver la zone moyenne à haute car le rendement du sorgho était meilleur qu'en zone basse, mais ils n'ont pas fait de distinction entre les cultures pures et celles associées, et le béref (citrullus vulgaris), troisième culture, est aujourd'hui remplacé par le melon et la pastèque. Ici, l'association sorghoniébé est majoritaire bien que les paysans préfèrent le sorgho, car ils connaissent les avantages du niébé : un cycle plus court, une levée plus rapide (c'est une graine nue), un gardiennage inutile lors de sa maturation, des feuilles fraîches consommées en sauce, et des résidus qui sont un fourrage apprécié du bétail (et plus riche en azote que les tiges de sorgho). Implanter du niébé, associé ou non au sorgho, est donc moins risqué, notamment en zone basse.

En 2018, 33,5 ha implantés (soit 7\%), dont 16 ha situés sous $360 \mathrm{~cm} \mathrm{NG}$, ont été abandonnés (classe «nul»); 437 ha, dont $75 \%$ situés au-dessus de $360 \mathrm{~cm} \mathrm{NG}$, ont produit une récolte, mais 245 ha étaient classés «faible» (Fig. 9b). La proportion de surface classée «forte» était plus grande audessus de $360 \mathrm{~cm} \mathrm{NG}$ qu'au-dessous. Tout cela confirme les remarques précédentes.

\subsection{Les productions de décrue en 2018}

Les mesures faites montrent des rendements en sorgho et niébé très variables selon la note de densité de végétation
(Tab. 1). L'analyse de variance indique un effet très significatif $(p<0,01 \%)$ de la note de végétation sur les rendements en grains et en fourrage du sorgho regroupé avec le niébé, mais pas de différence significative entre les notes «moyenne» et «forte». Par ailleurs, la production de melon et/ou de pastèque, très aléatoire, peut être une source de revenu complémentaire (jusqu'à $50000 \mathrm{FCFA} / \mathrm{ha}$ ). Les rendements de sorgho mesurés sont plus faibles que ceux indiqués par Boutillier et al. (1962), mais voisins de ceux indiqués par Saarnak (2003); ces travaux anciens et récents n'ont malheureusement pas précisé les rendements en fourrages.

Même si les producteurs déclarent auto-consommer la quasi-totalité de leur production, on peut estimer sa valeur économique. À la récolte, les grains de sorgho ou de niébé peuvent se vendre 400 à $500 \mathrm{FCFA} / \mathrm{kg}$, les tiges de sorgho $70 \mathrm{FCFA} / \mathrm{kg}$ et les cosses de niébé $300 \mathrm{FCFA} / \mathrm{kg}$ (les fanes de niébé sont aussi valorisées en fourrage, mais nous n'avons pas pu estimer les quantités produites). Ensuite, les prix augmentent fortement $(1200 \mathrm{FCFA} / \mathrm{kg}$ pour les grains en juin-juillet et jusqu'à $2000 \mathrm{FCFA} / \mathrm{kg}$ en octobre-novembre, à l'époque des semis de décrue). À la récolte, la valeur de la production d'une parcelle de décrue en 2018 a ainsi été estimée entre $78000 \mathrm{FCFA} /$ ha (végétation faible) et $245000 \mathrm{FCFA} / \mathrm{ha}$ (végétation moyenne ou forte), pour des coûts de production - essentiellement le coût des semences car la main-d'œuvre, sauf exception, est familiale - estimés à moins de 8000 FCFA/ ha. Sur ces bases, la production totale de la cuvette de Podor pour la décrue 2018 a été évaluée à 121 tonnes de grains et 117 tonnes de fourrage (hors fanes de niébé), pour une valeur totale de plus de 66 millions FCFA (hors melons et pastèques), soit 141000 FCFA par hectare implanté. Cette valeur est un peu plus faible que celle indiquée par Saarnak (2003), qui ne tient compte que des surfaces récoltées, mais plus élevée que celle utilisée dans le Schéma directeur d'aménagement et de gestion des eaux du fleuve Sénégal (SDAGE Sénégal; CSE et al., 2011). Ce produit brut est faible comparé à celui d'une culture irriguée, mais à l'inverse de celle-ci, il y a une production de fourrage de qualité et aucun risque financier, puisque le coût de production est très faible et n'oblige à aucun emprunt bancaire. Il peut aussi paraître faible au regard de la perte de production hydroélectrique que peut engendrer le soutien de crue par le barrage de Manantali (Bader et al., 2003). Les choix de gestion des ouvrages existants et futurs doivent néanmoins s'appuyer sur une étude approfondie des bénéfices, pas simplement économiques, qu'apportent à court et long termes les nombreux services agro-écosystémiques nés de l'inondation (cultures de décrue, mais aussi recharge des nappes, pêche, forêts...).

\section{Conclusion}

L'historique des crues à Podor montre que l'ampleur et la durée moyennes des crues étaient plus fortes avant 1976, mais le niveau du fleuve à l'étiage était alors $2 \mathrm{~m}$ plus bas. Dans la cuvette, l'inondation atteignait plus souvent certaines zones élevées impropres aux cultures et la période propice aux semis était plus courte et plus tardive.

Depuis 2003 et la mise en service des turbines hydroélectriques à Manantali, la superficie inondée exploitable médiane dans la cuvette de Podor est de 1132 ha, et nos 
Tableau 1. Productions moyennes dans un échantillon de 20 parcelles selon leur note de végétation (les rendements (a) et (b), d'une part, et (c) et (d), d'autre part, sont significativement différents au seuil 0,01\%).

Table 1. Average yields in a sample of 20 plots according to their vegetation score (yields [a] and [b] on the one hand, and [c] and [d] on the other hand, are significantly different at the $0.01 \%$ threshold).

\begin{tabular}{|c|c|c|c|c|}
\hline & & \multicolumn{3}{|c|}{ Densité de végétation } \\
\hline & & Faible & Moyen & Fort \\
\hline & $\mathrm{Nb}$ de parcelles & 8 & 5 & 7 \\
\hline \multirow[t]{2}{*}{ Sorgho (kg/ha) } & Grains & 56 & 242 & 287 \\
\hline & Tiges & 81 & 369 & 440 \\
\hline \multirow[t]{4}{*}{ Sorgho + Niébé (kg/ha) } & Grains & $144^{(\mathrm{a})}$ & $424^{(b)}$ & $455^{(\mathrm{b})}$ \\
\hline & & & & \\
\hline & Tiges et cosses & $108^{(\mathrm{c})}$ & $424^{(\mathrm{d})}$ & $490^{(\mathrm{d})}$ \\
\hline & & & & \\
\hline
\end{tabular}

observations permettent de faire l'hypothèse qu'environ $50 \%$ peuvent être exploités en cultures de décrue. Sur la base des résultats de la campagne 2018, cette superficie médiane exploitée pourrait produire 146 tonnes de grains pour l'alimentation humaine et 141 tonnes de fourrage pour le bétail. À l'échelle de la moyenne vallée, cette production n'est pas négligeable puisqu'après un hivernage 2017 défavorable au nord du Sénégal et l'absence de surface exploitable en décrue (Action contre la faim, 2017), les départements de Matam et Podor ont été déclarés proches d'une crise alimentaire pour les populations et les troupeaux (Sud Quotidien, 2018). Cette situation critique démontre que l'agriculture irriguée dans ces départements est encore insuffisante pour atténuer les aléas des productions pluviales et de décrue, qui occupent encore (au moins de manière complémentaire) une frange non négligeable de leur population.

L'agriculture de décrue est encore vitale pour l'alimentation des populations et des troupeaux en période de soudure, au moins à l'échelle locale. Actuellement, le barrage de Manantali contrôle $50 \%$ du débit du fleuve Sénégal, mais la construction du barrage de Gourbassi sur la Falémé est actée, et $70 \%$ du débit sera alors contrôlé. D'un côté, si l'agriculture irriguée doit remplacer celle de décrue, les cultures irriguées doivent se diversifier de manière à varier l'alimentation et produire des fourrages de qualité. On pourrait cultiver par exemple du sorgho et du niébé dans les périmètres irrigués rizicoles, notamment ceux qui risquent d'être inondés par la crue et qui ne sont donc exploités au mieux qu'une fois l'an. D'un autre côté, si le soutien de crue engendre un coût en pertes d'énergie électrique, il préserve non seulement des surfaces cultivées en décrue, mais aussi des écosystèmes et des nappes alluviales.

Remerciements. Cette étude a été pour partie financée par l'Agence française de développement (AFD) via son Programme de promotion du partenariat rizicole dans le Delta du fleuve Sénégal (3PRD), et le ministère de l'Europe et des Affaires étrangères via son Fonds de solidarité prioritaire AGRICORA. Les auteurs remercient Mamadou Wone et Ibra Kane, les producteurs de la cuvette de Podor, tout particulièrement Djénéba Wone et Oumar Watt, ainsi que les agents de la Société nationale d'aménagement et d'exploitation des terres du delta du fleuve Sénégal et des vallées du fleuve Sénégal et de la Falémé (SAED) de la délégation de Podor.

\section{Références}

Action contre la faim. 2017. [2017/10/10]. Disponible sur http:// sigsahel.info/wp-content/uploads/2017/10/Note-Alerte-Nord-Sene gal-ACF-Oct-2017.pdf.

Adams WM. 1993. Indigenous use of wetlands and sustainable development in West Africa. Geographical Journal 159(2): 209 218. DOI: $10.2307 / 3451412$.

Bader JC. 2014. Actualisation des résultats du POGR concernant le soutien de la crue du fleuve Sénégal par le barrage de Manantali. Paris (France): IRD, $48 \mathrm{p}$.

Bader JC, Lamagat JP, Guigen N. 2003. Gestion du barrage de Manantali sur le fleuve Sénégal: analyse quantitative d'un conflit d'objectifs. Hydrological Sciences Journal 48(4): 525-538. DOI: 10.1623/hysj.48.4.525.51415.

Bader JC, Belaud G, Lamagat JP, Ferret T, Vauchel P. 2017. Modélisation de propagation d'écoulement entre lits mineur et majeur sur les fleuves Sénégal et Niger. Hydrological Sciences Journal 62(3): 447-466. DOI: 10.1080/02626667.2016.1148815.

Boivin P, Brunet D, Gascuel-Odoux C, Zante P, Ndiaye JP. 1995. Les sols argileux de la région de Nianga-Podor: répartition, caractéristiques, aptitudes et risques de dégradation sous irrigation. In: Boivin $\mathrm{P}$ et al., eds. Nianga, Laboratoire de la Culture Irriguée: Atelier ORSTOM-ISRA, Saint-Louis 19-21 oct 1993. Paris (France) : ORSTOM, pp. 67-81.

Boutillier JL, Schmitz J. 1987. Gestion traditionnelle des terres (système de décrue/système pluvial) et transition vers l'irrigation : le cas de la vallée du Sénégal. In : Systèmes de production agricole en Afrique tropicale: 3. Système de production et de développement. Cahiers des sciences humaines 23(3/4): 533-554.

Boutillier JL, Cantrelle P, Causse J, Laurent C, N'Doye T. 1962. La moyenne vallée du Sénégal: étude socio-économique. Paris (France) : PUF, $368 \mathrm{p}$.

Bruckmann L. 2016. L'intégration des zones inondables dans la gestion de l'eau et le développement de l'irrigation d'une vallée fluviale sahélienne. Le cas des terres de décrue de la moyenne vallée du Sénégal. Thèse de doctorat. Paris (France): Université Paris Diderot, $581 \mathrm{p}$. 
Centre de suivi écologique (CSE), Compagnie d'aménagement des Coteaux de Gascogne (CACG) et Société du Canal de Provence (SCP). 2011. SDAGE du Fleuve Sénégal - Phase 3 - Schéma Directeur. Dakar (Sénégal)/Bruxelles (Belgique) : OMVS/Union européenne, 138 p.

Chantereau J, Cruz JF, Ratnadass A, Trouche G, Fliedel G. 2013. Le sorgho. Versailles (France)/Wageningen (Pays-Bas)/Gembloux (Belgique): Éditions Quae/CTA/Presses agronomiques de Gembloux, 20 p. DOI: 10.35690/978-2-7592-2062-5.

Comas J, Gómez MacPherson H. 2002. La culture du sorgho de décrue en Afrique de l'Ouest et du Centre. Madrid (Espagne)/Rome (Italie) : AECI/FAO, $247 \mathrm{p}$.

Jamin, JY. 1986. L'agriculture de décrue dans la vallée du fleuve Sénégal: les cultures traditionnelles du waalo et du falo. Note à l'attention des conseillers agricoles. Dakar (Sénégal)/Paris (France) : ISRA/CIRAD, $26 \mathrm{p}$.

JICA. 2013. https://www.jica.go.jp/senegal/french/activities/infra structure 03.html.

Lamagat JP. 2001. Programme d'optimisation de la gestion des réservoirs. Phase 3. Crue artificielle et cultures de décrue. Synthèse finale. Paris (France)/Dakar (Sénégal) : IRD/OMVS, 67 p.

Lericollais A, Schmitz J. 1984. La calebasse et la houe : techniques et outils des cultures de décrue dans la vallée du Sénégal. Cahiers ORSTOM, Série sciences humaines 20(3-4): 427-452.
Le Roy X. 2008. Le sorgho de décrue dans la vallée du Sénégal. In: Mollard E, Walter A, eds. Agricultures singulières. Paris (France) : IRD, pp. 33-38. ISBN 978-2-7099-1623-3.

Maymard J. 1957. Étude expérimentale des facteurs naturels influant sur les cultures de décrue : les essais de Guédé 1956-1957 : mission d'aménagement du Sénégal. Paris (France) : ORSTOM, 111 p. + annexes.

Maymard J. 1960. Études pédologiques dans la vallée alluviale du Sénégal. Ministère de l'Agriculture du Sénégal, Div. agronomique, bull. $122,38 \mathrm{p}$.

Saarnak NL. 2003. Flood recession agriculture in the Senegal River valley. Geografisk Tidsskrift 103(1): 99-113. DOI: 10.1080/ 00167223.2003.10649483.

SCP, CSE, CACG, Ginger. 2009. SDAGE du fleuve Sénégal. Phase 1. État des lieux et diagnostic, version finale, décembre 2009. Dakar (Sénégal): OMVS, 443 p.

Sidibé S, Williams TO, Kolavalli S. 2016. Flood Recession Agriculture for Food Security in Northern Ghana: Literature Review on Extent, Challenges, and Opportunities. GSSP Working Paper 42. Washington DC (USA): IFPRI, $18 \mathrm{p}$.

Sud Quotidien. 2018. [2018/04/25]. http://www.sudonline.sn/podormatam-ranerou-kanel-tambacounda-et-goudiry-menaces-par-la-cri se_a_39296.html.

Citation de l'article : Poussin J-C, Martin D, Bader J-C, Dia D, Seck SM, Ogilvie A. 2020. Variabilité agro-hydrologique des cultures de décrue. Une étude de cas dans la moyenne vallée du fleuve Sénégal. Cah. Agric. 29: 23. 